\title{
COVID-19, Autonomy, and the Inpatient Psychiatric Unit
}

\author{
Stephanie London ${ }^{1}$
}

Received: 19 May 2020 / Accepted: 8 September 2020/Published online: 23 September 2020

(C) Academic Psychiatry 2020

Jacob could not keep his mask on. The material felt harsh and irritating against his skin. He felt claustrophobic and found it difficult to breathe. Jacob is a young man with schizophrenia who was hospitalized involuntarily on our psychiatric unit, a medical psychiatric unit in a major urban hospital, at the peak of the COVID-19 crisis (his name has been changed to protect confidentiality). Jacob was one of the first patients I cared for on what happened to be my first inpatient psychiatry rotation as a doctor and psychiatry resident. It was also during this time that our hospital adopted new rules around COVID protection, including a requirement that all patients wear masks whenever they are outside their rooms. This is especially limiting to the psychiatric patient population, who are otherwise physically healthy and ambulatory and usually move freely throughout the psychiatric unit.

Jacob struggled with disorganized thinking and found it particularly difficult to comply with our hospital's mask policy. Even when Jacob would agree to wear his mask, it would inevitably end up ripped, tucked beneath his chin, or hanging off his ear entirely. Alongside nursing and occupational therapy, we tried different approaches to encourage Jacob to wear his mask appropriately. Efforts to make the mask more comfortable were not successful. Extensive conversations about the importance of mask wearing, while met with some understanding and motivation, could not overcome his discomfort.

Our team discussed the risks posed to others by allowing Jacob not to wear a mask with the potential harms of damaging our therapeutic relationship and potentially triggering agitation. Psychiatric units are especially susceptible to viral outbreaks given patient challenges with personal hygiene as well as frequent interactions with others in day rooms and during groups [1]. Mask wearing is not just a question of personal autonomy, but also a choice that puts others at risk. The choice by one

Stephanie London

slondon1@partners.org

1 Massachusetts General Hospital/McLean Adult Psychiatry Residency Program, Boston, MA, USA patient not to wear a mask jeopardizes the safety of all other patients on the unit, an already vulnerable population. On the other hand, even in the community where mask wearing is strongly encouraged, it is rarely enforced. We often protect individual autonomy to make medical decisions, even when those decisions could affect the health of others, such as with vaccines. As a practical matter, if a patient on a psychiatric unit refuses to wear a mask, the only way to keep them out of common spaces is through seclusion or restraint, which has proven to be traumatic and have serious repercussions on patient well-being.

Ultimately, our team continued to ask Jacob to stay in his room and only come into common spaces if he was wearing a mask. This led to near constant verbal redirection, encouragement to use as needed medications, and eventually escalated to Jacob becoming frustrated and severely agitated, necessitating the use of physical restraints. Jacob had otherwise been in good behavioral control throughout his hospitalization, and not required restraints for any other reason.

As an early psychiatry trainee, I began learning how and when to use restraints and seclusion for the first time amidst the COVID pandemic. During the time we treated Jacob, the Massachusetts Department of Mental Health (DMH) had not offered clear guidance on how to enforce mask policies on psychiatric units. More specific DMH policies were outlined subsequently allowing for restraints or seclusion to be used to maintain isolation for patients known to be COVID positive or at risk for being positive without confirmatory testing (Commonwealth of Massachusetts DMH Bulletin \#20-03). Even these guidelines fail to capture the challenges of mask policies on psychiatric units, an added limitation to an already highly restrictive environment. In Jacob's case, mask wearing became a daily discussion on morning rounds, distracting from other aspects of his care. Moreover, it was the conflict around mask wearing that fueled Jacob's episodes of verbal and physical agitation, and that eventually necessitated restraints to maintain safety.

The debate over COVID protection policies exaggerates the tension between autonomy and safety that is always present on a psychiatric unit. While we may understand the reason 
or necessity for restrictive measures, we often forget how unfamiliar and abrasive these restrictions can feel to those experiencing them. In some ways, it was easy for me to identify with Jacob's frustrations. I was also adjusting to new restrictions in my daily routine. I was asked to space myself from co-workers, often moving to makeshift workrooms in order to maintain distance. I learned to account for extra time in the morning so that I could disinfect my work station at the beginning of each shift. Like Jacob, I struggled with the discomfort of wearing a mask all day, especially during the long hours in the hospital during intern year, when moments of relief are few and far between.

Even outside of the hospital, almost all of us have experienced limitations to our autonomy. We have been told where to go, what to wear, who to be close to, often with inconsistent messaging from governmental and medical agencies alike. We've struggled to adhere to these restrictions, to limit ourselves in ways we did not think were negotiable.

Now more than ever, we have an opportunity to understand the experience of our patients. Limitations to autonomy are not unique to psychiatry; patients across disciplines experience significant loss of control and power when they are hospitalized or sick. During this crisis, many have learned what it means to be quarantined indoors, even to a single room or bed, a daily reality for hospitalized patients. As psychiatrists, we are tasked with the responsibility of caring for patients who have had their civil liberties taken away, telling them to live temporarily in extremely restrictive environments. In the case of Jacob, I still wonder if we were too forceful, if we could have been more flexible or lenient. While I don't think there is a clear answer, my own experiences during the COVID pandemic have given me more empathy for Jacob, and a better understanding of the profound impact of losing autonomy.

Acknowledgements Dr. Judith Restrepo for editing and conceptual guidance.

Compliance with Ethical Standards The author states that there are no conflicts of interest.

Disclosures Names in this piece have been changed to protect confidentiality.

\section{Reference}

1. Gessen M. Why psychiatric wards are uniquely vulnerable to the coronavirus. The New Yorkers. 2020.

Publisher's Note Springer Nature remains neutral with regard to jurisdictional claims in published maps and institutional affiliations. 